\title{
Entropy Generation Analysis in a Bioinspired Microchannel
}

\author{
Prasenjit Dey, Sandip K. Saha \\ Department of Mechanical Engineering, Indian Institute of Technology Bombay \\ Mumbai 400076, India \\ prasenjit.dey@iitb.ac.in; sandip.saha@iitb.ac.in
}

\begin{abstract}
In this study, numerical analysis of entropy generation in a novel bio-inspired surface developed microchannel is presented. A three dimensional conjugate heat transfer model is developed to analyse the entropy generation in the microchannel of hydraulic diameter of $187.5 \mu \mathrm{m}$ and length of $20 \mathrm{~mm}$. The working fluid is Deionized water and the flow is maintained for three different Reynolds number $(R e)=250,650$ and 1050. The novel surface is inspired by fish scales and patterned on the bottom surface of the microchannel at which a constant heat flux of $100 \mathrm{~W} / \mathrm{cm}^{2}$ is applied. The entropy generation rate is inversely proportional to the values of inclination angle $(\alpha)$. A maximum of $42 \%$ reduction in entropy generation rate is observed in the MC_A10 $\left(\alpha=10^{\circ}\right)$ microchannel at $R e=1050$ compared to the plain microchannel. The MC_A10 microchannel has the least value of augmentation entropy generation number, $\eta_{s}=$ 0.58 at $\operatorname{Re}=1050$.
\end{abstract}

Keywords: Microchannel, Bioinspired Surface, Heat Transfer, Entropy Generation.

\section{Introduction}

After the introduction of microchannel heat sink by Tuckerman and Pease [1], the fluid flow and heat transfer analysis in microchannel receive immense attention by the numerous researchers due to its application in electronics cooling, laser equipment, medical instruments, and micro heat exchanger systems [2]. As the microchannel affects the cooling performance of electronic devices, hence it is pertinent to design a microchannel for better hydrothermal performance by reducing the irreversibility caused by the fluid friction and temperature difference between the fluid and surface. The importance of the heat transfer enhancement mechanism of different shaped microchannels can be evaluated by comparing the entropy generation rate. Hence, entropy generation analysis is important to optimize the second law performance of these microcscale channels.

The characteristics of the irreversibility of flow and heat transfer process were evaluated by entropy generation mechanism for the first time by Bejan [3]. The entropy generation and transfer were analysed by Chen [4] in a microchannel for different thermal boundary conditions. It was observed that the temperature gradient in the stream wise direction was the prime reason to develop the local entropy generation rate. It was also found that the optimum fluid temperature distribution due to the exchanging of heat by the fluid to the surrounding was a linear one. An analytical approach was used by Abbassi [5] to solve the forced convection problem and also to investigate the entropy generation in a microchannel. A dimensionless form of local and average entropy generation was derived by the calculated velocity and temperature fields. The total entropy generation rate was formulated by Yazdi et al. [6] by integrating the local entropy generation in a microchannel with a constant heat flux boundary condition for different values of slip coefficient. It was observed that the total entropy and fluid friction can be sufficiently reduced by the slip flow circumstances. The effect of viscous dissipation on the entropy generation was studied by Hung [7]. The entropy generation was calculated in a single phase liquid flow circular microchannel supplied by uniform heat flux and it was observed that there was significant effect of viscous dissipation on entropy generation and should not be neglected. The heat transfer irreversibility in different geometric structured microchannels was studied by different researchers by applying the entropy generation minimization technique [8,9]. Also, several researchers have extensively studied the well-known bioinspired (lotus leaf, rice leaf, rose petals etc.) surface in microchannel to enhance single phase heat transfer performance however there was no availability of entropy generation characteristics in these types of bioinspired microchannels [10 - 12].

Hence, it can be noted that different techniques are existed for augmenting the heat transfer rate in microchannels and one of these techniques is bioinspired surface. However, it is important to analyse the entropy generation rate in order to 
justify the advantage of these heat transfer enhancement techniques. Therefore, the scope of the present study is to perform a numerical analysis in a novel bioinspired surface motivated by the fish scales to quantify its merit on the heat transfer rate enhancement by evaluating the entropy generation rate considering three different values of $\operatorname{Re}=250,650$, and 1050 in three different geometric fish scale microchannels and also in a plain microchannel.

\section{Numerical Model}

The numerical analysis is carried out on a microchannel of hydraulic diameter, $D_{h}=193.5 \mu \mathrm{m}$ made of copper in which the bottom surface is enriched with bio-inspired fish scale surface as shown in Fig. 1. The width $(W)$ and height $(H)$ of the microchannel is $3000 \mu \mathrm{m}$ and $100 \mu \mathrm{m}$, respectively. The geometry of the fish scale is defined by the slant height $\left(\mathrm{S}_{\mathrm{f}}\right)$, width of scale $\left(W_{f}\right)$, horizontal gap $\left(H_{f}\right)$, vertical height $\left(V_{f}\right)$ and inclination angle $(\alpha)$ as depicted in Fig. 1. A total eight numbers of fish scales are generated on the bottom surface of the channel. The distance between the two stream-wise fish scales and centre distance between the two fish scales in the $z$ direction is 4 and $0.75 \mathrm{~mm}$, respectively.

Four types of microchannels are designed for the present numerical model. These are plain channel (no fish scales at the bottom surface, MC_P), MC_A1 $\left(S_{f} / D_{h}=1.50, W_{f} / D_{h}=1.07, H_{f} / D_{h}=1.07, V_{f} / D_{h}=1.60\right.$, and $\left.\alpha=1^{\circ}\right), \mathrm{MC}_{-} \mathrm{V}\left(S_{f} / D_{h}=1.50\right.$, $W_{f} / D_{h}=1.07, H_{f} / D_{h}=1.07, V_{f} / D_{h}=2.13$, and $\left.\alpha=5^{\circ}\right)$, and MC_A10 $\left(S_{f} / D_{h}=1.50, W_{f} / D_{h}=1.07, H_{f} / D_{h}=1.07, V_{f} / D_{h}=1.60\right.$, and $\alpha=10^{\circ}$ ). A three dimensional conjugate heat transfer model is used for the fluid flow and heat transfer study to investigate the entropy generation in the microchannel. The working fluid is the Deionised water and the steady state laminar flow condition is considered for three values of $R e=250,650$ and 1050. Few assumptions are considered in the present numerical model such as the flow is Newtonian incompressible flow, no variation of the fluid properties with the considered range of temperature, and no significant losses due to radiation and natural convection. Hence, the generalized governing equation considering single domain approach for both the fluid and solid can be written as,

Conservation of mass:

$$
\nabla \cdot V=0
$$

Conservation of momentum:

$$
\rho(V . \nabla \mathrm{V})=-\nabla P+\mu \nabla^{2} V
$$

Conservation of energy:

$$
\rho c_{p}(V . \nabla \mathrm{T})=k \nabla^{2} T
$$

The following boundary conditions are applied in the present computational domain. At $x=0$, the uniform velocity inlet is imposed at a constant ambient temperature and is expressed as,

$$
u(y)=U_{\infty} \text { and } T=T_{a m b}
$$

At $x=L$, the pressure outlet condition is applied where pressure gradient is zero and is written as,

$$
\nabla P=0
$$

A constant heat flux, $q^{\prime \prime}=100 \mathrm{~W} / \mathrm{cm}^{2}$ on the bottom surface of the microchannel whereas the right side is considered as the symmetry plane. Adiabatic wall boundary condition is applied on the left and top surfaces of the channel and is expressed as,

$$
u=0 ; \frac{\partial T}{\partial z}=\frac{\partial T}{\partial y}=0
$$

For the conjugate heat transfer modelling, the thermal conditions at the solid-liquid interface are set as, 


$$
\begin{aligned}
-\left.k_{l} \frac{\partial T_{l}}{\partial n}\right|_{\text {interface }} & =-\left.k_{s} \frac{\partial T_{w}}{\partial n}\right|_{\text {interface }} \\
\left.T_{l}\right|_{\text {interface }} & =\left.T_{w}\right|_{\text {interface }}
\end{aligned}
$$
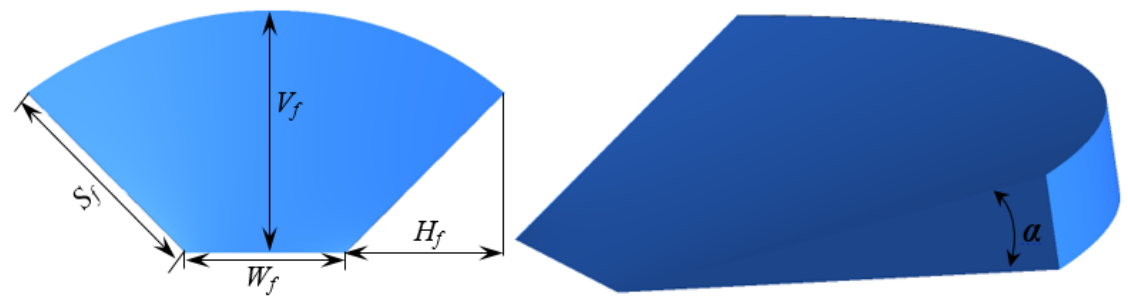

(a)

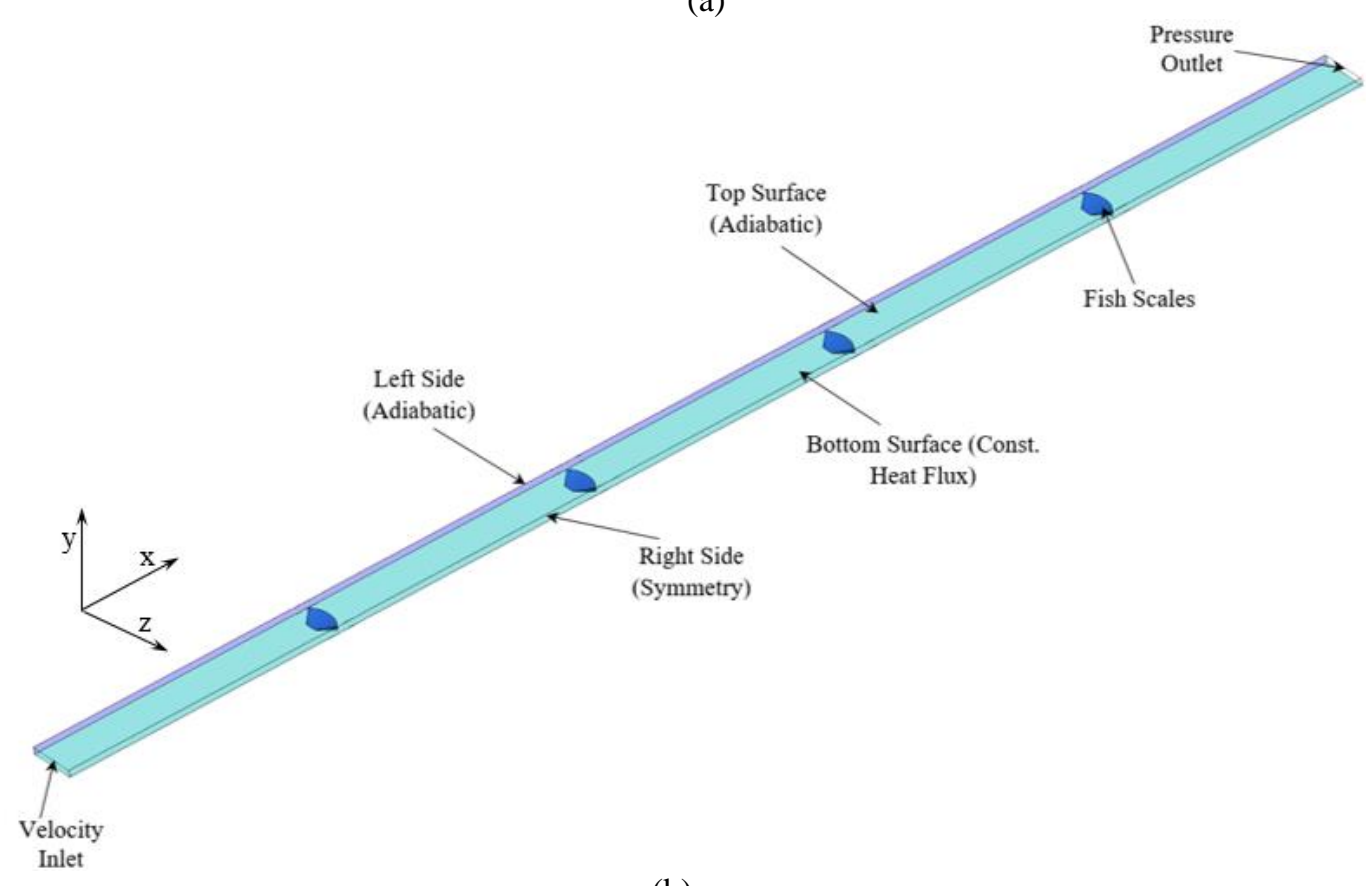

(b)

Fig. 1: (a) Geometry of the bio-inspired fish scales and (b) present numerical domain with boundary condition.

The main objective of the present numerical study is to analyse the flow and heat transfer irreversibility in the novel microchannel, hence the entropy generation method is implemented in this analysis. The total entropy generation rate in the microchannel is the addition of the entropy generation rate due to the friction loss and entropy generation rate due to the heat transfer caused by temperature difference. Hence, the total volume entropy generation rate can be expressed as [3, 8,9],

$$
S_{g e n}^{\prime \prime \prime}=S_{g e n, \Delta P}^{\prime \prime \prime}+S_{g e n, \Delta T}^{\prime \prime \prime}
$$

where, $S_{g e n, \Delta P}^{\prime \prime \prime}$ is the volume entropy generation due to friction by the flow and $S_{g e n, \Delta T}^{\prime \prime \prime}$ is the volume entropy generation rate due to temperature difference and both can be expressed as,

$$
S_{g e n, \Delta P}^{\prime \prime \prime}=\frac{\mu}{T_{l}}\left[2\left\{\left(\frac{\partial u}{\partial x}\right)^{2}+\left(\frac{\partial v}{\partial y}\right)^{2}+\left(\frac{\partial w}{\partial z}\right)^{2}\right\}+\left(\frac{\partial u}{\partial y}+\frac{\partial v}{\partial x}\right)^{2}+\left(\frac{\partial u}{\partial z}+\frac{\partial w}{\partial x}\right)^{2}\left(\frac{\partial v}{\partial z}+\frac{\partial w}{\partial y}\right)^{2}\right]
$$




$$
S_{g e n, \Delta T}^{\prime \prime \prime}=\frac{k_{l}}{T_{l}}\left\{\left(\frac{\partial T}{\partial x}\right)^{2}+\left(\frac{\partial T}{\partial y}\right)^{2}+\left(\frac{\partial T}{\partial z}\right)^{2}\right\}
$$

where, $u, v$ and $w$ are velocity components in the $x, y$ and $z$ directions respectively.

If these above volume entropy generation rate is further integrated within the computational fluid domain then the entropy generation rate due to fluid friction $\left(\dot{S}_{g e n, \Delta P}\right)$, due to temperature difference $\left(\dot{S}_{g e n, \Delta T}\right)$ and total entropy generation rate $\left(\dot{S}_{g e n}\right)$ becomes,

$$
\begin{gathered}
\dot{S}_{g e n}=\dot{S}_{g e n, \Delta P}+\dot{S}_{g e n, \Delta T} \\
\dot{S}_{g e n}=\frac{\dot{m} \Delta P}{\rho T_{l}}+\frac{q^{\prime \prime} A_{b}\left(T_{w}-T_{f}\right)}{T_{w} T_{l}}
\end{gathered}
$$

For measuring the efficacy of reducing the irreversibility of the present novel microchannel compared to that of the plain channel, augmentation entropy generation number $\left(\eta_{s}\right)$ [3] is adopted, which is the ratio of entropy generation rate of novel bioinspired microchannel $\left(\dot{S}_{g e n}\right)$ to the entropy generation rate of plain microchannel $\left(\dot{S}_{g e n, 0}\right)$ and expressed as,

$$
\eta_{s}=\frac{\dot{S}_{g e n}}{\dot{S}_{g e n, 0}}
$$

The Finite Volume Method (FVM) based commercial solver ANSYS Fluent V16 is used to solve the governing equations in the present numerical analysis.

A thorough grid independence study is conducted to find the optimized minimum number of grids within the computational domain for less computational time with significant accuracy in the outcomes. Hexahedral type of control volumes is generated within the whole domain and a total number 901500 of cells is chosen by considering the error of less than $0.25 \%$ in total entropy generation rate between the two different number of cells occupied microchannels. An experimental study is conducted on the MC_A10 microchannel for three values of $R e=250$, 650, and 1050 . The pressure, surface temperature and fluid temperature are measured by using two absolute pressure transducers and ten T-Type thermocouples. The pressure transducers are located at the inlet and outlet plenums to measure the fluid inlet and outlet pressures, whereas eight thermocouples are inserted in the copper surface block below the bottom wall of the microchannel to measure the surface temperature and two other thermocouples are located at the inlet and outlet of the channel to measure the fluid inlet and outlet temperatures. Based on the measured pressure and temperatures, the entropy generation rate due to fluid friction $\left(\dot{S}_{g e n, \Delta P}\right)$ and temperature difference $\left(\dot{S}_{g e n, \Delta T}\right)$ and total entropy generation rate $\left(\dot{S}_{g e n}\right)$ are calculated and summarized in Table 1 along with the corresponding numerical data. It is observed that the maximum error between the experimental and numerical total entropy generation rates $\left(\dot{S}_{g e n}\right)$ is $2.52 \%$.

Table 1: Percentage error between the experimental and numerical entropy generation rates.

\begin{tabular}{|c|c|c|c|c|}
\hline $\boldsymbol{R e}$ & & $\dot{\boldsymbol{S}}_{\boldsymbol{g e n}, \Delta \boldsymbol{P}}$ & $\dot{\boldsymbol{S}}_{\boldsymbol{g e n}, \Delta \boldsymbol{T}}$ & $\dot{\boldsymbol{S}}_{\boldsymbol{g e n}}$ \\
\hline \multirow{3}{*}{250} & Experimental & $2.61 \times 10^{-5}$ & $2.05 \times 10^{-2}$ & $2.06 \times 10^{-2}$ \\
\cline { 2 - 5 } & Numerical & $2.52 \times 10^{-5}$ & $2.00 \times 10^{-2}$ & $2.00 \times 10^{-2}$ \\
\cline { 2 - 5 } & Error (\%) & 3.45 & 2.58 & 2.52 \\
\hline \multirow{3}{*}{650} & Experimental & $2.12 \times 10^{-4}$ & $1.42 \times 10^{-2}$ & $1.44 \times 10^{-2}$ \\
\cline { 2 - 5 } & Numerical & $2.08 \times 10^{-4}$ & $1.39 \times 10^{-2}$ & $1.41 \times 10^{-2}$ \\
\cline { 2 - 5 } & Error (\%) & 1.82 & 2.01 & 1.96 \\
\hline \multirow{3}{*}{1050} & Experimental & $6.50 \times 10^{-4}$ & $1.14 \times 10^{-2}$ & $1.20 \times 10^{-2}$ \\
\cline { 2 - 5 } & Numerical & $6.39 \times 10^{-4}$ & $1.12 \times 10^{-2}$ & $1.19 \times 10^{-2}$ \\
\cline { 2 - 5 } & Error (\%) & 1.74 & 1.58 & 1.56 \\
\hline
\end{tabular}




\section{Results and Discussions}

\subsection{Velocity distribution}

The fluid flow behaviour within the channel and the distribution of temperature in the fluid are characterized by the velocity and temperature profile for three values of $R e=250,650$ and 1050 at three different sections viz. first section is at the leading edge of the first fish scale (F1), second is at the trailing edge of the first fish scale (F2) and third is at the recirculation zone behind the first fish scale $(\mathrm{F} 3)$.

The velocity gradient along the $y$-axis for three different sections as mentioned earlier is shown in Fig. 2. It is observed from Fig. 2 (a) that the flow is fully developed at the leading edge of the first fish scale for each microchannel where the maximum dimensionless velocity is found as 1.5 at the centre of the channel. For a constant value of $R e$, there is no significant variation in the velocity profile for each microchannels. However, the velocity profile is slightly reduced for higher values of $R e$ which is due to the more frictional shear forces generated within the microchannel produced by the resistance to flow at high $R e$ values. When the fluid passes over the fish scales and flows up to the trailing edge, the parabolic velocity profile is affected in MC_V and MC_A10. However, the MC_P channel shows the fully developed velocity profile as can be seen in Fig. 2 (b). The maximum velocity is found in MC_A10 $\left(\alpha=10^{\circ}\right)$ due to the less passage area of the flow caused by the higher inclination angle of fish scales, whereas the velocity profile is remained parabolic in MC_A1 $\left(\alpha=1^{\circ}\right)$ channel as the flow is very less affected by the smaller inclination angle of the fish scales. The intermediate behaviour is observed in MC_V. This disturbance in the flow and the higher velocity in MC_V and MC_A10 enhances the mixing of cold and hot fluids which consequently enhances the heat transfer rate. The maximum non-dimensional velocity is found as 2.08 in MC_A10 at $R e=250$. At the third section, it is observed from the Fig. 2 (c) that the velocity distribution is same for MC_P and MC_A1, however the velocity distribution in $\mathrm{MC} \_\mathrm{V}$ and MC_A10 are more chaotic compared to that at the trailing edge of the fish scales. Also, a strong recirculation zone can be observed (where the velocity is $\leq 0$ ) at $R e=650$ and 1050 in MC_A10 channel. The disturbance in the velocity profile for MC_V and MC_A10 increases with increasing $R e$. In this zone, the maximum non-dimensional velocity is found as 1.80 in MC_A10 at $R e=650$. The drop in the maximum velocity from 2.08 to 1.80 is due to the expansion in the flow passage after the trailing edge of the fish scales.

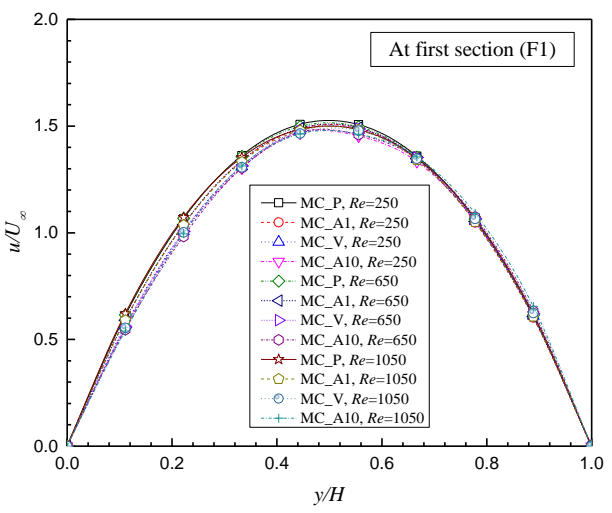

(a)

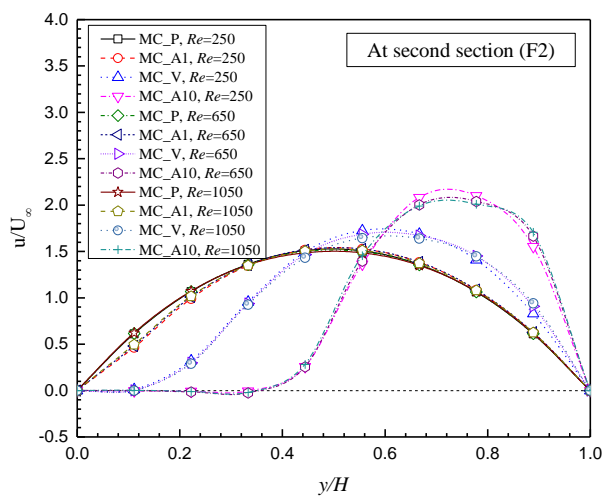

(b)

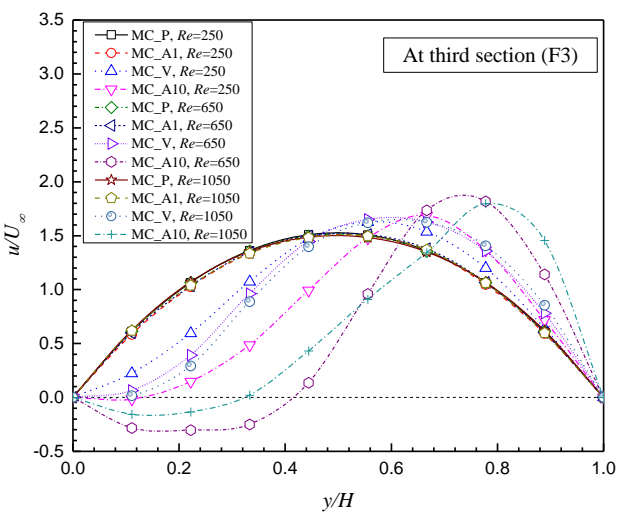

(c)

Fig. 2: Non-dimensional velocity profile along the height of the microchannel at three different sections. 


\subsection{Temperature Distribution}

The non-dimensional temperature $(\theta)$ distribution throughout the microchannel height ( $y$ direction) for three values of $R e=250,650$ and 1050 at three different sections as mentioned earlier is depicted in Fig. 3. It is perceived from Fig. 3 (a) that at the leading edge of the first fish scale, the value of $\theta$ is high at the bottom surface of the channel $(y / H=0)$ and gradually decreases up to $\theta=0$ towards the top surface $(y / H=1)$. The value of dimensionless temperature decreases with the increase in $R e$, which is mainly due to higher interaction between cold fluid and hot surface at higher value of $R e$ and consequently takes away more heat from the hot surface. For every considered $R e$, the increase in dimensionless temperature is observed in every bio-inspired microchannels. With the increase of heat transfer area in bioinspired microchannels, more heat is transferred to the fluid. Hence, the bulk mean fluid temperature in bioinspired channels is more than that in the plain channel. As surface temperature is low for bioinspired surface, the dimensionless temperature is higher at the core region. This overall characteristic augments the thermal performance and it is observed that the dimensionless temperature at $y / H=0$ (bottom surface), is always lesser in MC_A1, MC_V and MC_A10 compared to that of MC_P at every Re. The value of $\theta$ at the bottom surface at $R e=250$ and 1050 for MC_A10 is 0.88 and 0.46 , respectively, whereas the respective value of $\theta$ for MC_P is 1 and 0.59. At the trailing edge of the first fish scale as shown in Fig. 3(b), the same temperature distribution characteristics are observed, whereas there is a fluctuation in $\theta$ observed in MC_A10 at $R e=650$ and 1050, which is caused by the fluctuations in velocity distribution as discussed earlier. This disturbance in temperature profile in MC_A10 is more at the recirculation zone as shown in Fig. 3(c). As there is more fluctuation in the velocity within the recirculation zone behind the fish scales, more disturbance in the dimensionless temperature is observed. The value of $\theta$ at the bottom surface at $R e=250$ and 1050 for MC_A10 is 0.90 and 0.27 respectively whereas the corresponding value of $\theta$ for MC_P is 1 and 0.59 .

\subsection{Entropy Generation Rate}

The variation in the entropy generation rate $\left(\dot{S}_{g e n}\right)$ with $R e$ is shown in Fig. 4. As discussed earlier the entropy generation rate depends on the $\dot{S}_{g e n, \Delta P}$ and $\dot{S}_{g e n, \Delta T}$, and it is observed that the $\dot{S}_{g e n}$ highly depends on the $\dot{S}_{g e n, \Delta T}$ (not shown here). The value of $\dot{S}_{g e n}$ decreases rapidly with $R e$ as the $\dot{S}_{g e n, \Delta T}$ decreases asymptotically with $R e$. This decrement in $\dot{S}_{g e n, \Delta T}$ with $R e$ is mainly because of the less temperature difference between the fluid and surface at higher $R e$. The least value of the $\dot{S}_{g e n}$ is observed in MC_A10, which is 0.02, 0.014 and 0.012, whereas the maximum value is found in the plain channel, which is $0.025,0.022$, and 0.020 for $R e=250,650$ and 1050 respectively. When the inclination angle $(\alpha)$ of the fish scales is increased, the thermal performance is also enhanced by virtue of which the value of $\dot{S}_{g e n}$ is lesser at higher value of $\alpha$.

The variation of the augmentation entropy generation number $\left(\eta_{s}\right)$ with $R e$ is depicted in Fig. 4(b). According to the definition, lesser the value of $\eta_{s}$ than 1 , better is the microchannel to reduce the fluid flow and heat transfer reversibility. It is observed that the present novel bioinspired microchannel performs better than the plain microchannel for each considered $R e$. The value of $\eta_{s}$ for every bioinspired microchannel decreases rapidly with the increase in $R e$ by virtue of the enhancement in the heat transfer rate with minor increase in pressure drop. The MC_A10 $\left(\alpha=10^{\circ}\right)$ microchannel is found as the best bioinspired microchannel for reducing the fluid flow and heat transfer reversibility for each value of $R e=250,650$ and 1050 . As discussed earlier, the higher value of inclination angle of fish scales increases the fluid velocity within the channel and also enhances the mixing of fluid with the surfaces, which accordingly augments the thermal performance of the channel than that of the lower inclination angle of the fish scales. The least value of $\eta_{s}$ in MC_A10 is found as $0.79,0.63$, and 0.58 for $R e=250,650$ and 1050, respectively. 


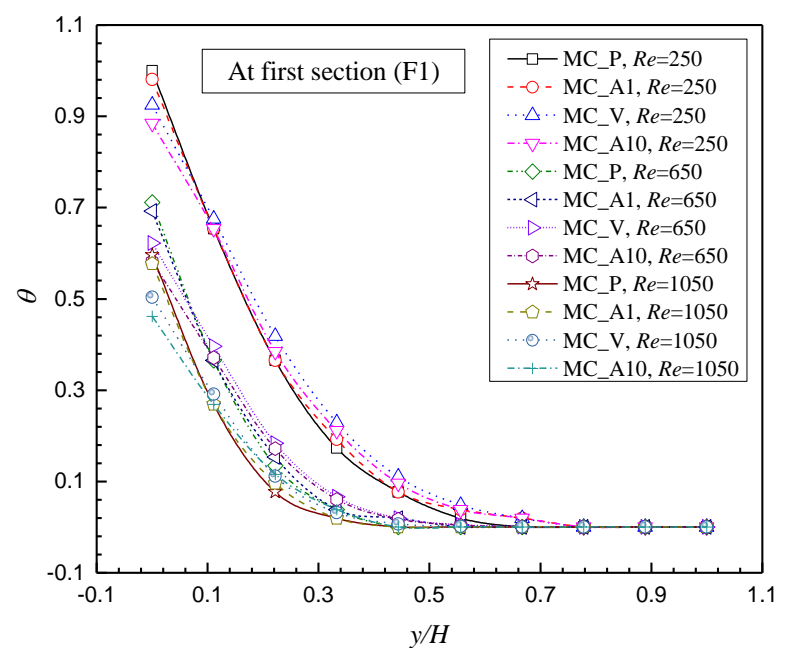

(a)

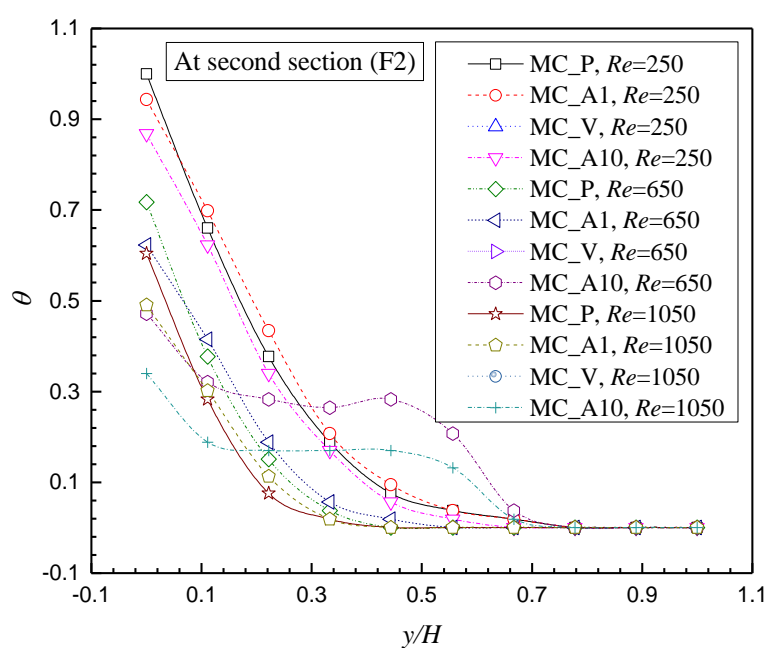

(b)

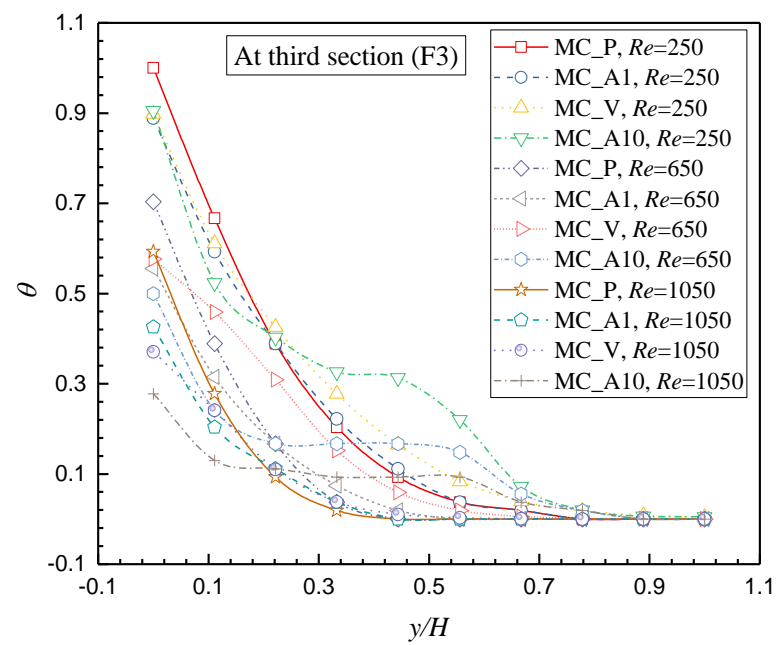

(c)

Fig. 3: Non-dimensional temperature profile along the height of the microchannel at three different sections.

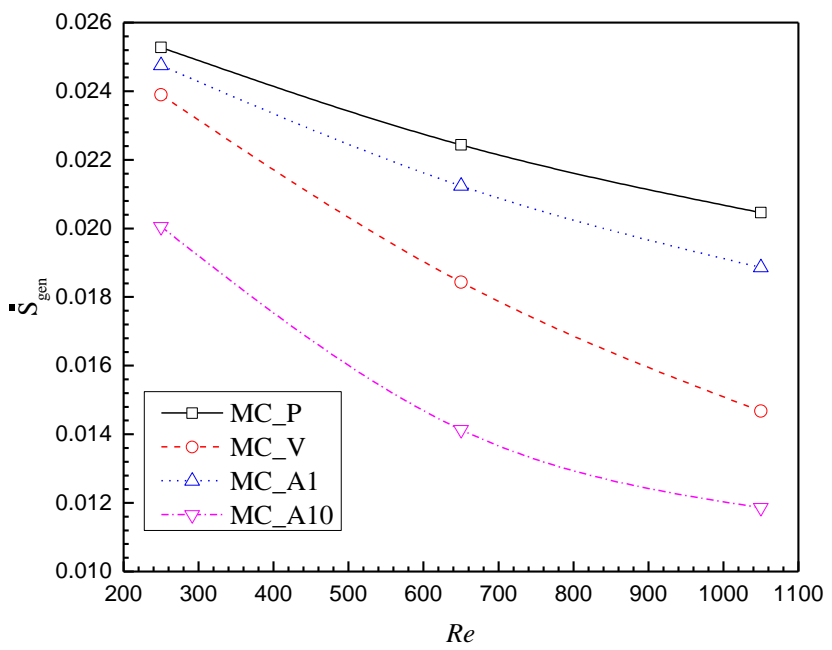

(a)

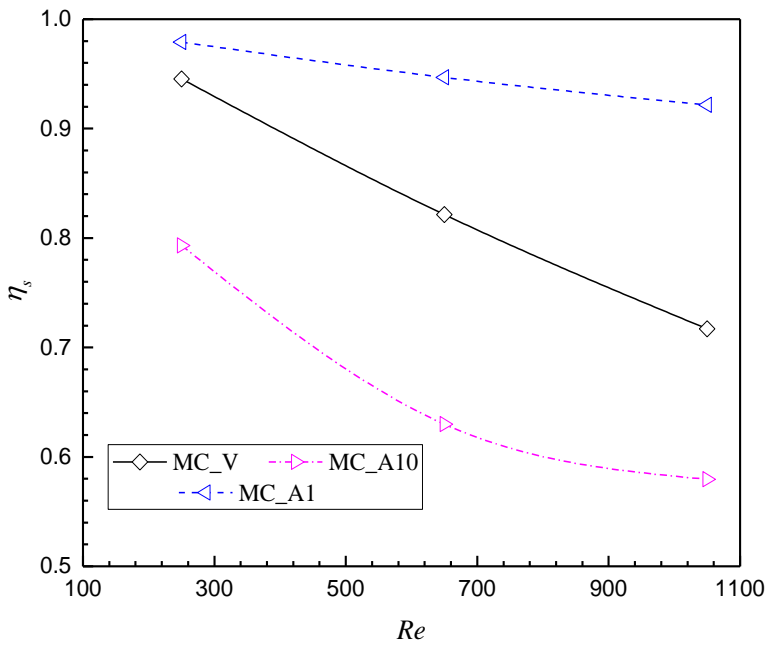

(b)

Fig. 4: Variation of (a) $\dot{S}_{g e n}$ and (b) $\eta_{s}$ with $R e$. 


\section{Conclusions}

In this paper, a numerical analysis is conducted on a novel bioinspired microchannel to analyse the entropy generation rate for quantifying the fluid flow and heat transfer irreversibility characteristics. Three types of bioinspired surface enhanced microchannels are considered along with the plain channels and three values of $R e=250,650$ and 1050 are considered. It is observed that the fully developed velocity profile exists at the leading edge of the first fish scale in the bioinspired microchannel for every considered $R e$. When the inclination angle $(\alpha)$ of the fish scales is increased from $1^{\circ}\left(\mathrm{MC} \_\mathrm{A} 1\right)$ to the $5^{\circ}\left(\mathrm{MC} \_\mathrm{V}\right)$ or $10^{\circ}$ (MC_A10), the velocity profile is more disturbed at the leading edge of the fish scales and as well as at the recirculation zone. This disturbance in the velocity distributions creates disruption in the dimensionless temperature profile. It is found that the surface temperature of the present novel microchannel is less than that of the plain channel and this value is inversely proportional to the inclination angle of the fish scales. It is also observed that the entropy generation rate is very less in the present novel microchannels and least value is found in the MC_A10 microchannel. The entropy generation rate is also inversely proportional to the inclination angle $(\alpha)$ of the fish scales. The maximum of $42 \%$ reduction in entropy generation rate is observed in the MC_A10 microchannel at $R e=1050$ compared to the plain microchannel. Further, augmentation entropy generation number $\left(\eta_{s}\right)$ is calculated to measure the efficacy of the reduction in the fluid flow and heat transfer irreversibility of the present microchannel compared with the plain microchannel. It is observed that the MC_A10 microchannel has the least value of $\eta_{s}=0.58$ at $R e=1050$.

\section{Nomenclature}

\begin{tabular}{|c|c|c|c|}
\hline$D_{h}$ & Hydraulic diameter $\left(\frac{2 W H}{(W+H)}\right), \mathrm{m}$ & $R e$ & Reynolds number $\left(\frac{\rho U_{\infty} D_{h}}{\mu}\right)$ \\
\hline$c_{p}$ & Specific heat (J/kg.K) & $A_{b}$ & Bottom surface Area, $\mathrm{W} \times \mathrm{L}\left(\mathrm{m}^{2}\right)$ \\
\hline$k$ & Thermal conductivity (W/m.K) & $p$ & Pressure, bar \\
\hline$T$ & Temperature & $Q$ & Heat transfer rate $(W)$ \\
\hline$q^{\prime \prime}$ & Heat flux, $\mathrm{W} / \mathrm{m}^{2}$ & & \\
\hline \multicolumn{4}{|c|}{ Greek Symbol } \\
\hline$\rho$ & Density $\left(\mathrm{kg} / \mathrm{m}^{3}\right)$ & $\mu$ & Dynamic Viscosity $(\mathrm{kg} / \mathrm{m} . \mathrm{s})$ \\
\hline$\theta$ & Non-dimensional temperature & & \\
\hline \multicolumn{4}{|c|}{ Subscript } \\
\hline$l$ & Fluid & $w$ & Wall surface \\
\hline$\infty$ & Free stream & $a m b$ & Ambient condition \\
\hline
\end{tabular}

\section{References}

[1] D. B. Tuckerman, R. Pease, "High-performance heat sinking for VLSI. Electron Device," Lett IEEE, vol. 2, no. 5, pp. 126-9, 1981.

[2] A. Ebrahimi, R. Farhad, A. Sabaghan, R. Ehsan, "Heat transfer and entropy generation in a microchannel with longitudinal vortex generators using nanofluids," Energy, Vol. 101, pp. 190-201, 2016.

[3] A. Bejan, Entropy Generation Minimization. CRC press, Florida, 1996.

[4] K. Chen, "Second-law analysis and optimization of microchannel flows subjected to different thermal boundary conditions," Int J Energ Res., vol. 29, pp. 249-263, 2005.

[5] H. Abbassi, "Entropy generation analysis in a uniformly heated microchannel heat sink," Energy, vol. 32, pp. 19321947, 2007.

[6] M. H. Yazdi, S. Abdullah, I. Hashim, K. Sopian, A. Zaharim, "Entropy generation analysis of liquid fluid past embedded open parallel microchannels within the surface," Eur J Sci Res., vol. 28, no. 3, pp. 462-470, 2009.

[7] Y. M Hung, "A comparative study of viscous dissipation effect on entropy generation in single-phase liquid flow in microchannels," Int. J. Therm. Sci., vol. 48, pp. 1026-1035, 2009.

[8] Y. L. Zhai, G. D. Xia, X. F. Liu, Y. F. Li. "Heat transfer in the microchannels with fan-shaped reentrant cavities and different ribs based on field synergy principle and entropy generation analysis," International journal of heat and mass transfer, vol. 68, pp. 224-233, 2014.

[9] Y. F. Li, G. D. Xia, D. D. Ma, Y. T. Jia, J. Wang, "Characteristics of laminar flow and heat transfer in microchannel heat sink with triangular cavities and rectangular ribs," International Journal of Heat and Mass Transfer, vol. 98, pp. 17-28, 2016. 
[10] Y. Cheng, J. Xu, Y. Sui. "Numerical study on drag reduction and heat transfer enhancement in microchannels with superhydrophobic surfaces for electronic cooling," Applied Thermal Engineering, vol. 88, pp. 71-81, 2015.

[11] F. Y. Lv, P. Zhang, "Drag reduction and heat transfer characteristics of water flow through the tubes with superhydrophobic surfaces," Energy Conversion and Management, vol. 113, pp. 165-176, 2016.

[12] D. Maynes, K. Jeffs, B. Woolford, B.W. Webb, "Laminar flow in a microchannel with hydrophobic surface patterned microribs oriented parallel to the flow direction," Phys Fluids., vol. 19, pp. 093603-12, 2007. 